九州大学学術情報リポジトリ

Kyushu University Institutional Repository

\title{
Synthesis of Small Lipid Nanoparticles Using an Inkjet Mixing System Aiming to Reduce Drug Loss
}

Inohara, Kota

Department of Chemical Engineering, Kyoto University

Asano, Shusaku

Institute for Materials Chemistry and Engineering, Kyushu University

Maki, Taisuke

Department of Chemical Engineering, Kyoto University

Mae, Kazuhiro

Department of Chemical Engineering, Kyoto University

http://hdl. hand le. net/2324/2545007

出版情報 : Chemical Engineering and Technology. 42 (10)，pp.2061-2066，2019-09-24. Wiley バージョン：

権利関係 : 


\author{
Mr. Kota Inohara ${ }^{1}$ \\ Dr. Shusaku Asano² \\ Dr. Taisuke Maki ${ }^{1, *}$ \\ Dr. Kazuhiro Mae ${ }^{1}$
}

\title{
Synthesis of small lipid nanoparticles using an inkjet mixing system aiming to reduce drug loss
}

\begin{abstract}
An inkjet mixing system was investigated to synthesize lipid nanoparticles (LNPs) by mixing lipid ethanol and aqueous saline solutions. The system was employed to help minimize drug loss. The quick mixing of the droplets enabled the synthesis of small LNPs with reducing the excess amount of saline solution which would contain drugs. The system also features minimal usage of reagent. Small LNPs were successfully synthesized with less saline solution than previous studies using micromixers. A high relative velocity and moderate values of the impact parameter were necessary for successful mixing. A faster droplet should have collided with the upper or horizontal level of a slower droplet to promote mixing. A horizontal spin of the coalesced droplets was favored rather than a vertical spin.
\end{abstract}

\section{Keywords}

collision, droplets, inkjet, lipid nanoparticle, mixing

\section{Addresses/Affiliations}

${ }^{1}$ Department of Chemical Engineering, Graduate School of Engineering, Kyoto University, Kyotodaigaku Katsura, Nishikyo-ku, Kyoto 615-8510, Japan

${ }^{2}$ Institute for Materials Chemistry and Engineering, Kyushu University, 6-1, Kasuga Koen, Kasuga, Fukuoka 816-8580, Japan

Email ${ }^{*}$ corresponding author: tmaki@cheme.kyoto-u.ac.jp

\section{Text of the contribution}

\section{Introduction}

Drug delivery systems (DDSs) have been designed to improve the effectiveness and safety of drug administration by controlling pharmacokinetics and biodistribution of drugs [1, 2]. Many kinds of drug carriers for DDS have been developed: lipid nanoparticles (or liposomes [3, 4]), lipid microspheres [5], polymeric micelles [6, 7], and gold nanoparticles [8]. Nanometer-sized drug carriers have received much attention because 
of their great potential. Lipid nanoparticles (LNPs) are considered one of the most useful and suitable nanometer-sized carriers [9]. LNPs are composed of lipid bilayers, which are the same components as cell walls. Therefore, LNPs have desirable features as DDS carriers. They have a long circulation time in the blood and are non-damaging to cells. Drugs using LNPs as carriers have already been clinically approved in many countries. When utilizing LNPs as a DDS carrier for an anticancer drug, the diameter of the LNPs is the most important factor in biodistribution [10, 11], because 20 - $100 \mathrm{~nm}$ LNP can accumulate tumor tissue due to the enhanced permeability and retention effect. In addition, as LNPs become smaller, the circulation time in the blood will be prolonged, and more LNPs will reach the tumor site and maximize their effect. The reticuloendothelial system hardly removes small LNPs. Chen et al. investigated circulation lifetime of small interfering RNA encapsulated in LNPs coated with (R)-2,3-bis(stearyloxy)propyl-1-(methoxy poly(ethylene glycol)2000 carbamate. The smallest LNP with a diameter of $30 \mathrm{~nm}$ showed the longest circulation time [12].

For synthesizing 20 - $100 \mathrm{~nm}$ LNPs, the rapid mixing method using micromixers has attracted attention recently in addition to the most commonly employed sonication method [13-15]. Micromixers enable rapid mixing down to the submillisecond scale by reducing the diffusion length [16, 17]. Rapid mixing provides ideal homogeneous conditions for synthesizing small and monodispersed particles in a bottom-up manner. The rapid mixing method achieves the synthesis of LNPs by feeding an aqueous saline solution and a lipid ethanol solution into a micromixer. Maeki et al. assumed the mechanism was as follows [18]. When the ethanol concentration decreases, lipid molecules self-assemble and form bilayered phospholipid fragments (BPFs). BPFs transform into LNPs to decrease the surface energy. Quick dilution of ethanol prevents the growth of BPFs. The fast mixing and large volume ratio of the aqueous solution to the ethanol solution (VR) result in small LNPs. Maeki et al. synthesized quite small empty LNPs under high VR conditions by using micromixers [18-20] e.g., the LNP size was approximately $40 \mathrm{~nm}$ at VR $=3$ and approximately $20 \mathrm{~nm}$ at $\mathrm{VR}=9$. Note that synthesizing empty LNPs without drugs in usual for basic study on the evolution of LNPs [21, 22].

Despite the advantage of a higher VR for making smaller LNPs, operations with lower VR conditions are desirable when a remote loading method is not applicable. The remote loading method encloses drugs in carriers through $\mathrm{pH}$ or concentration gradients [23, 24]. However, a few drugs are accessible. Other drugs must be dissolved in an aqueous saline solution before mixing [25, 26]. In this case, most of the drug remains in the solution and is not encapsulated by the LNPs. Intensifying the mixing would help to reduce the VR and loss of drugs. An increase in the flow rate is a practical solution for improving the mixing performance. However, the amount of solution required for each experiment also increases with the flow rate. Screening of a drug using LNPs as a DDS carrier requires sets of drugs encapsulated LNPs in small amounts [27, 28]. Further improvement in the mixing system to synthesize only the required amount of LNPs with a small VR would enhance the applicability of the rapid mixing method.

This study focused on an inkjet mixing system as an alternative to the conventional micromixer. The inkjet nozzle can eject droplets of controlled sizes with high reproducibility. In recent years, inkjet technology is spreading various fields beyond conventional image printing. For example, Gupta et al. developed programmable release capsules with 3D-printing technology using inkjet nozzles [29]. Manipulation of droplets by inkjet nozzles enabled precise spatiotemporal control over chemical and biomolecular gradients. Petukhov 
et al., conducted reactive inkjet printing of conducting patterns made by nickel and copper [30]. They first printed metal salt ink to the substrate and then printed reducing ink to form a seed layer. Precise manipulation of small droplets by inkjet technology would also be beneficial for LNPs synthesis. However, small LNP synthesis requires fast mixing, which would be difficult on the substrate. The inkjet mixing system, which uses two nozzles to mix droplets in the air, would be suitable for LNP synthesis. The system used in this study is shown in Fig. 1. Two nozzles facing each other eject droplets with diameters of 40-100 $\mu \mathrm{m}$, or with a volume of approximately 30-500 pL. Two ejected droplets collide and coalesce in air, as shown in Fig. 1b. The rapid mixing concept by colliding droplets was confirmed in the previous study. Takano et al. examined the collision between ethanol droplets and water droplets with identical sizes and reported mixing times faster than a millisecond [31]. Mixing behavior can be adjusted by changing the droplet velocity and the nozzle position, rather than changing the flow rate as with a conventional micromixer. Furthermore, minimal reagent consumption can easily be achieved by designating the number of droplets. Davis et al. reported that an inkjet mixing system actually consumed smaller amounts of reagents than micromixers [32]. The inkjet mixing system is suitable for synthesizing small LNPs only the required amount for minimizing loss of drugs. In the synthesis of LNPs, the collision between droplets with different physical properties and diameters, i.e., large droplets of aqueous saline solution and small droplets of lipid ethanol solution has to be controlled. In this paper, the ideal operating conditions for the inkjet mixing system based on a detailed analysis of droplet behaviors, such as coalescence, separation, deformation, and spin are discussed.

Methods

2.1 Equipment and Materials. The inkjet mixing system was an inkjet kit, IJK-200W (Microjet Corp., Nagano, Japan). The nozzles used for droplet collision were 1-nozzle inkjet heads, IJHB-100 (Microjet Corp.). Photographs of droplet collision were taken by a high-sensitivity black/white camera, WAT-902H ULTIMATE (Watec Co., Ltd., Yamagata, Japan). Dynamic light scattering (DLS) was performed using a nanoparticle analyzer SZ-100 (HORIBA Ltd., Kyoto, Japan). The cell used for DLS analysis was either a quartz black microcell, NR-4511-01 (AS ONE Corp., Osaka, Japan), or a quartz cell, S10-UV-10 (GL Sciences Inc., Tokyo, Japan).

2.2 Binary droplets collision by an inkjet mixing system. Two solutions were prepared. 1Palmitoyl-2-oleoyl-sn-glycero-3-phosphocholine (POPC) (NOF Corp., Tokyo, Japan) was dissolved in ethanol (WAKO Pure Chemical Industries Ltd., Osaka, Japan), and a $10 \mathrm{mg} \mathrm{mL}^{-1}$ lipid ethanol solution was obtained. Sodium chloride (WAKO Pure Chemical Industries) was dissolved in water, and a $0.9 \mathrm{wt} \%$ saline solution was obtained. Inkjet nozzles ejected lipid ethanol solution droplets and saline solution droplets, and they collided. The ejection velocities of the lipid ethanol solution droplets and saline solution droplets are shown in Tab. 1, and the velocities were measured from photographs. The diameter of the lipid ethanol solution droplet, $d_{\text {lipid, }}$ and saline solution droplet, $d_{\text {saline }}$ were also measured from the photographs, and the volume ratio (VR, aqueous saline solution/lipid ethanol solution) was calculated. The measured $d_{\text {lipid }}=42 \mu \mathrm{m}$, and $d_{\text {saline }}=56 \mu \mathrm{m}$, and the 
calculated VR was 2.4 in all experiments. Similarly, the major axis diameter was measured from photographs taken every $3.5 \mu \mathrm{s}$. The native software for controlling inkjet mixing system (HCS+, CAP) has an auto calibration function, which indicated the scale bar for images. Weber number (We) was calculated by Eq. (1), where $\rho, u, d$, and $\sigma$ are the density, relative velocity of the two droplets, coalesced droplet diameter, and interface tension, respectively. The following parameters were utilized : $\rho=962 \mathrm{~kg} \mathrm{~m}^{-3}$ and $\sigma=0.0357 \mathrm{~N} \mathrm{~m}^{-1}$, which were the density and interfacial force of aqueous ethanol solution $\left(\mathrm{VR}=2.4,20^{\circ} \mathrm{C}\right)$; the relative velocity, $u$, was calculated using the velocities of droplets in Tab. 1; the diameter of the coalesced droplets, $d$, was calculated from the sum of the volumes of the droplets before the coalescence, calculated from $d_{\text {lipid }}$ and $d_{\text {saline }}$. The head-on collision and the off-center collision in the up and down direction or depth direction were achieved by changing the position of the nozzle up and down or backward by several tens of micrometers. The impact parameter, $B$, was calculated by Eq. (2), where $b$ and $d_{\text {lipid }}$ are the distance between two droplets and the lipid ethanol solution droplet diameter, respectively. In the inkjet mixing system, the impact parameter, $B$, had two dimensions because the nozzles could be moved either horizontally or vertically. These dimensions were expressed by the indices y and z. $B_{\mathrm{y}}$ denoted the up and down direction and $B_{\mathrm{z}}$ denoted the depth direction perpendicular to this page. The ranges of $B_{\mathrm{y}}$ and $B_{\mathrm{z}}$ were $-1 \leq B_{\mathrm{y}} \leq 1$, and $0 \leq B_{\mathrm{z}} \leq 1$. The distance between the two nozzles was fixed at $2.5 \mathrm{~mm}$. The strobe camera cycle was $3.5 \mu \mathrm{s}$. Photographs showing the change of the droplet after collision were made by capturing a series of droplet collision photographs and superimposing them. In other words, photographs did not follow the movement of one droplet, but each was different droplet. Droplets were ejected at a rate of 500 count $\mathrm{s}^{-1}$. The droplets were collected in a vial under the two nozzles. The falling distance was fixed at $5 \mathrm{~cm}$ in all experiments. The residence time of the droplets in the air was tens of milliseconds, which was much longer than the expected mixing time of the collided droplets. The droplet collision experiment was conducted at room temperature and was windless for each experiment. After collection of the coalesced droplets, the resulting LNP solution in the vial was analyzed with DLS. The viscosity of the product solution was calculated to be $2.435 \mathrm{mPa} \cdot \mathrm{s}$ based on the previous report [33]. The refractive index of the synthesized LNPs was calculated by using equations reported by Matsuzaki et al. [34].

$$
\begin{aligned}
& W e=\frac{\rho d u^{2}}{\sigma} \\
& B=\frac{b}{d_{\text {lipid }}}
\end{aligned}
$$

\section{3}

\section{Results and Discussion}

3.1 Effects of relative velocity of two droplets. The relationship between the relative velocity of two droplets and the LNP diameter was investigated with fixing $B_{\mathrm{y}}$ and $B_{\mathrm{z}}$ to be 0 . Two droplets were collided only in a head-on collision. Fig. 2a shows the LNP diameter in number-weighted mean particle size vs. the We number. In a head-on collision, two colliding droplets coalesced in the region of $W e<50$. And the droplets separated in the region of We $\geq 50$. Qian et al. reported that colliding droplets were also separated by a high inertial force as the We number increased [35]. In the coalescence region of $W e<50$, a larger We number, which 
meant larger inertial force, made the synthesized LNP diameters smaller. Fig. $2 \mathrm{~b}$ shows the particle size distribution of the synthesized LNPs at each We number. The average particle size of LNPs gradually became smaller as the We number increased, but the size distributions of LNPs were not significantly changed. Fig. 2c shows the deformation behavior of droplets that collided at each We number. The left side droplet was composed of the lipid ethanol solution, and the right side was composed of the saline solution. Under all conditions, the coalesced droplets showed expansion and contraction behaviors. When the We number was small, the major axis diameter of the coalesced droplets was short. For instance, that diameter was $115 \mu \mathrm{m}$ at $W e=20$. The deformation of the coalesced droplets was not intense. When the We number increased, the major axis diameter became longer. The diameter was $127 \mu \mathrm{m}$ at $W e=42$. The deformation of the coalesced droplet was more intense. From these results, a large deformation by a high inertial force improved mixing, resulting in the synthesis of smaller LNPs. Relative velocities as high as possible would be important for synthesizing small LNPs.

3.2 Effects of impact parameter. The effects of impact parameter $B$ were investigated with fixing relative velocity of two droplets to be $6.36 \mathrm{~m} \mathrm{~s}^{-1}$. Fig. 3a shows the experimental conditions and whether colliding droplets coalesced or separated. Observations had been conducted for more than 1 hour for each point. The behavior of coalescence/separation was stable for each point. Red circles imply successful coalescence, and black crosses imply separation of the droplets. The We number was fixed at 69 with a lipid ethanol solution droplet velocity of $4.0 \mathrm{~m} \mathrm{~s}^{-1}$ and a saline solution droplet velocity of $5.0 \mathrm{~m} \mathrm{~s}^{-1}$. Just as with the results in the previous section, the droplets separated after a head-on collision, at such a high We number. The droplets coalesced at off-center collisions, as shown in i, iii, iv, v, and vi in Fig. 3. According to a previous study, offcenter conditions were effective at reducing the shock of collisions, by converting the shock into rotational energy [36]. On the other hand, a further increase in $B$ caused droplet separation. The deformation behaviors of the droplets after collision is shown in Fig. $3 \mathrm{~b}$ with the major axis diameters of the coalesced droplets and the synthesized LNP diameters for each condition. The deformation behaviors of the droplets and the LNP diameters changed with different impact parameters. When the impact parameter was changed in the up and down direction, the coalesced droplets showed vertical spins. In condition i, coalesced droplets showed counterclockwise vertical spin and condition iii, they showed clockwise vertical spin. When impact parameter was changed in depth direction, they showed horizontal spins. In condition v, coalesced droplets showed horizontal spin. In condition iv and vi, they showed spins combining vertical and horizontal surfaces. In conditions iii and v, the major axis diameters were equal. However, the LNP diameters were different. The same applies to condition i and iv. Factors other than the major axis diameters of droplets would change the LNP diameters.

The difference between condition iii, vi and i, iv, v can be attributed to the velocity difference of the two droplets. The mixing behavior differed based on whether the higher velocity saline solution droplets collided with the lipid ethanol solution droplets at negative $\left(B_{\mathrm{y}}<0\right.$, condition iii and vi) or positive $\left(B_{\mathrm{y}} \geq 0\right.$, condition $\mathrm{i}$, iv, and v) impact parameters. Fig. 4 shows a model image describing the phenomena. The model image was considered based on the shape evolution of the droplets shown in Fig. 3b. When $B_{\mathrm{y}}<0$, the faster saline solution 
droplet escaped from the lipid ethanol solution droplet, hereafter, this collision is called an "escaping collision". When the saline solution escaped from the lipid ethanol solution after colliding, mixing does not occur quickly. The saline solution area and lipid ethanol solution area were divided. Then, the two solutions were pulled each other because of the surface tension. Two solutions were mixed after these movements. The LNPs synthesized in conditions iii and vi were large. On the other hand, when $B_{y} \geq 0$, the saline solution droplet coated the lipid ethanol solution droplet. The better contact of solutions promoted rapid mixing. As a result, small LNPs would be synthesized. From these discussions, escaping collisions should be avoided.

An additional factor lied in the size differences in conditions $\mathrm{i}$, iv and $\mathrm{v}$ would be the direction of spin. Vertical spin of the coalesced droplet was observed in the condition i. Horizontal spins were observed in the conditions iv and v. It could be considered that horizontal spin promoted mixing rather than the vertical spin did.

The desirable operating conditions of the inkjet mixing system were summarized as follows: relative velocity is high, the droplet collision is not an escaping collision condition and the direction of the spin is horizontal. On conditions iv and v, the small LNPs (the diameter was under $30 \mathrm{~nm}$ at number-weighted mean particle size) were synthesized at a low volume ratio, $\mathrm{VR}=2.4$, in other words low consumption of saline solution. The synthesis of small LNPs with low consumption of saline solution was achieved.

\section{$4 \quad$ Conclusions}

LNPs were synthesized by using an inkjet mixing system. By changing the relative velocity of two droplets, the LNP diameters decreased when the relative velocity increased. The mixing of the two solutions was facilitated by the high inertial force. Not only the value of the impact parameters but also the velocity difference between the lipid ethanol and saline solutions was crucial. Off-center collisions were effective at preventing separation of the merged droplets. Avoiding escaping collisions was desirable for synthesizing smaller LNPs because mixing started promptly. The horizontal spin of the coalesced droplets seemed to promote the mixing of the two droplets. The inkjet mixing system could synthesize small LNPs with enabling low VR conditions and minimal consumption of samples, which would help to minimize loss of drugs in the DDS applications.

\section{Acknowledgements}

This work was supported by JSPS KAKENHI Grant numbers JP25220913 and JP26289295.

The authors declare that they have no competing interests.

\section{Symbols used}

B $\quad[-]$

the impact parameter

$b \quad[\mathrm{~m}]$

the distance between two droplets 


\begin{tabular}{|c|c|c|}
\hline$d$ & {$[\mathrm{~m}]$} & coalesced droplet diameter \\
\hline$d_{\text {lipid }}$ & {$[\mathrm{m}]$} & the lipid ethanol solution droplet diameter \\
\hline$d_{\text {saline }}$ & {$[\mathrm{m}]$} & the aqueous saline solution droplet diameter \\
\hline$u$ & {$\left[\mathrm{~m} \mathrm{~s}^{-1}\right]$} & the relative velocity of the two droplets \\
\hline We & {$[-]$} & Weber number \\
\hline
\end{tabular}

\section{Greek letter}

$\begin{array}{lll}\mu & {[\mathrm{mPa} \mathrm{s}]} & \text { the viscosity of the coalesced droplets } \\ \rho & {\left[\mathrm{kg} \mathrm{m}^{-3}\right]} & \text { the density of the coalesced droplet } \\ \sigma & {\left[\mathrm{N} \mathrm{m}^{-1}\right]} & \text { interface tension of the coalesced droplet }\end{array}$

\begin{tabular}{ll}
\multicolumn{2}{l}{ Abbreviations } \\
BPF & bilayered phospholipid fragment \\
DDS & drug delivery system \\
DLS & dynamic light scattering \\
LNP & lipid nanoparticle \\
VR & volume ratio
\end{tabular}

\section{References}

[1] T. M. Allen, P. R. Cullis, Science 2004, 303, 1818-1822. DOI: 10.1126/science.1095833

[2] H. Maeda, J. Wu, T. Sawa, Y. Matsumura, K. Hori, J. Control. Release 2000, 65, 271-284. DOI: 10.1016/S0168-3659(99)00248-5

[3] V. P. Torchilin, Nat. Rev. Drug Discov. 2005, 4, 145-160. DOI: 10.1038/nrd1632

[4] M. Ferrari, Nat. Rev. Cancer 2005, 5, 161-171. DOI: 10.1038/nrc1566

[5] Y. Mizushima, Y. Shoji, T. Kato, M. Fukushima, S. Kurozumi, J. Pharm. Pharmacol. 1986, 38, 132134. DOI: 10.1111/j.2042-7158.1986.tb04527.x

[6] M. Hrubý, Č. Koňák, K. Ulbrich, J. Control. Release 2005, 103, 137-148. DOI: 10.1016/j.jconrel.2004.11.017

[7] K. Kataoka, A. Harada, Y. Nagasaki, Adv. Drug Deliv. Rev. 2001, 47, 113-131. DOI: 10.1016/S0169409X(00)00124-1

[8] P. Ghosh, G. Han, M. De, C. K. Kim, V. M. Rotello, Adv. Drug Deliv. Rev. 2008, 60, 1307-1315. DOI: 10.1016/j.addr.2008.03.016

[9] K. Mäder, W. Mehnert, Adv. Drug Deliv. Rev. 2001, 47, 165-196. DOI: 10.1016/S0169409X(01)00105-3

[10] C. W. Perrault SD, Walkey C, Jennings T, Fischer HC, Nano Lett. 2009, 9, 1909-1915. DOI: 10.1021/nl900031y 
[11] C. Link, F. Alexis, E. Pridgen, L. K. Molnar, Mol. Pharm. 2008, 5, 505-515. DOI: $10.1155 / 2014 / 417305$

[12] S. Chen, Y. Yi, C. Tam, P. J. C. Lin, M. M. H. Sung, Y. K. Tam, P. R. Cullis, J. Control. Release 2016, 235, 236-244. DOI: 10.1016/j.jconrel.2016.05.059

[13] I. V. Zhigaltsev, N. Belliveau, I. Hafez, A. K. K. Leung, J. Huft, C. Hansen, P. R. Cullis, Langmuir 2012, 28, 3633-3640. DOI: 10.1021/la204833h

[14] C. H. Huang, Biochemistry 1969, 8, 344-352. DOI: 10.1021/bi00829a048

[15] D. Fleury, J. M. Walker, Liposomes, 2nd ed., Springer New York, New York, 2017.

[16] S. Asano, S. Yamada, T. Maki, Y. Muranaka, K. Mae, React. Chem. Eng. 2017, 2, 830-841. DOI: 10.1039/c7re00051k

[17] S. Asano, S. Yatabe, T. Maki, K. Mae, Org. Process Res. Dev. in press. DOI: 10.1021/acs.oprd.8b00356

[18] M. Maeki, Y. Fujishima, Y. Sato, T. Yasui, N. Kaji, A. Ishida, H. Tani, Y. Baba, H. Harashima, M. Tokeshi, PLoS One 2017, 12, e0187962. DOI: 10.1371/journal.pone.0187962

[19] M. Maeki, T. Saito, Y. Sato, T. Yasui, N. Kaji, A. Ishida, H. Tani, Y. Baba, H. Harashima, M. Tokeshi, RSC Adv. 2015, 5, 46181-46185. DOI: 10.1039/C5RA04690D

[20] N. Kimura, M. Maeki, Y. Sato, Y. Note, A. Ishida, H. Tani, H. Harashima, M. Tokeshi, ACS Omega 2018, 3, 5044-5051. DOI: 10.1021/acsomega.8b00341

[21] A. Jahn, S. M. Stavis, J. S. Hong, W. N. Vreeland, D. L. Devoe, M. Gaitan, ACS Nano 2010, 4, 20772087. DOI: $10.1021 / \mathrm{nn} 901676 \mathrm{x}$

[22] J. M. Zook, W. N. Vreeland, Soft Matter 2010, 6, 1352-1360. DOI: 10.1039/b923299k

[23] X. Li, D. J. Hirsh, D. Cabral-Lilly, A. Zirkel, S. M. Gruner, A. S. Janoff, W. R. Perkins, Biochim. Biophys. Acta - Biomembr. 1998, 1415, 23-40. DOI: 10.1016/S0005-2736(98)00175-8

[24] Y. C. Barenholz, J. Control. Release 2012, 160, 117-134. DOI: 10.1016/j.jconrel.2012.03.020

[25] Y. Barenholz, Curr. Opin. Colloid Interface Sci. 2001, 6, 66-77. DOI: 10.1016/S1359-0294(00)00090$\mathrm{X}$

[26] Y. Motokui, T. Wada, T. Isoda, S. Ichikawa, T. Kuroiwa, WO2010110116A1, 2010.

[27] Y. Sato, Y. Note, M. Maeki, N. Kaji, Y. Baba, M. Tokeshi, H. Harashima, J. Control. Release 2016, 229, 48-57. DOI: 10.1016/j.jconrel.2016.03.019

[28] S. M. Park, J. M. Cha, J. Nam, M. S. Kim, S.-J. Park, E. S. Park, H. Lee, H. R. Kim, PLoS One 2014, 9, e103116. DOI: 10.1371/journal.pone.0103116

[29] M. K. Gupta, F. Meng, B. N. Johnson, Y. K. Kong, L. Tian, Y. Yeh, N. Masters, S. Singamaneni, M. C. McAlpine, Nano Lett. 2015, 15, 5321-5329. DOI: 10.1021/acs.nanolett.5b01688

[30] D. I. Petukhov, M. Kirikova, A. A. Bessonov, M. A. Bailey, Mater. Lett. 2014, 132, 302-306. DOI: 10.1016/j.matlet.2014.06.109

[31] Y. Takano, S. Kikkawa, T. Suzuki, J. Y. Kohno, J. Phys. Chem. B 2015, 119, 7062-7067. DOI: 10.1021/acs.jpcb.5b03233 
[32] R. D. Davis, M. I. Jacobs, F. A. Houle, K. R. Wilson, Anal. Chem. 2017, 89, 12494-12501. DOI: 10.1021/acs.analchem.7b03601

[33] The Chemical Society of Japan, Kagaku Binran (in Japanese), 5th ed., Maruzen Publishing, Tokyo 2012.

[34] K. Matsuzaki, O. Murase, K. Sugishita, S. Yoneyama, K. Akada, M. Ueha, A. Nakamura, S. Kobayashi, Biochim. Biophys. Acta - Biomembr. 2000, 1467, 219-226. DOI: 10.1016/S0005-2736(00)00223-6

[35] J. Qian, C. K. Law, J. Fluid Mech. 1997, 331, 59-80. DOI: 10.1017/S0022112096003722

[36] M. Orme, Prog. Energy Combust. Sci. 1997, 23, 65-79. DOI: 10.1016/S0360-1285(97)00005-1 


\section{Tables with Headings}

Table 1. The velocities of the ejected droplets. The velocities were changed by adjusting the inkjet mixing system, the voltage applied to the piezo of the nozzle, and the 1st, pause, and 2nd pulse parameters. We number was calculated by using Eq. (1).

\begin{tabular}{llll}
\hline Conditions & $\begin{array}{l}\text { Lipid ethanol } \\
\text { solution }\left[\mathrm{m} \mathrm{s}^{-1}\right]\end{array}$ & $\begin{array}{l}\text { Aqueous saline } \\
\text { solution }\left[\mathbf{m ~ s}^{-1}\right]\end{array}$ & We number [-] \\
\hline 1 & 2.3 & 2.6 & 20 \\
2 & 2.9 & 2.9 & 29 \\
3 & 4.0 & 3.0 & 42 \\
4 & 4.0 & 4.0 & 54 \\
5 & 4.0 & 5.0 & 69 \\
\hline
\end{tabular}




\section{Figure legends}

Figure 1. Images of an inkjet mixing system. (a) The illustration of an inkjet mixing system. The nozzle diameter is $100 \mu \mathrm{m}$ and ejects 40-100 $\mu \mathrm{m}$ droplets at a rate of 30-2000 droplets per second. (b) A photograph of droplet collision taken by a high-sensitivity black/white camera. Nozzle 1 ejects ethanol, and nozzle 2 ejects water. The interval of the strobe was $14 \mu \mathrm{s}$.

Figure 2. Results for changing relative velocities. (a) The relationship between the LNP diameter and the We number. The LNP diameter is the mean diameter analyzed by DLS. The relative velocity $u$ corresponds with the $W e$ number. In the region of $W e<50$, the two droplets coalesced. In the region of $W e \geq 50$, they separated. (b) The particle size distribution of synthesized LNPs for each We number. (c) The photographs show the deformation behavior of droplet collisions. These photos of droplets were taken every $14 \mu$.

Figure 3. Changing the impact parameter. (a) Whether colliding droplet coalesced or separated on each impact parameter. (b) The deformation behavior of coalesced droplets under each condition. Major axis diameter and LNP diameter are attached. For all conditions, $W e=69$ (the relative velocity was approximately $6.36 \mathrm{~m} \mathrm{~s}^{-1}$ ) and the volume ratio VR was 2.4. In addition, each experiment was conducted at room temperature in a windless setting.

Figure 4. The model image of the mixing of lipid ethanol and saline solutions. The yellow area refers to the lipid ethanol solution, the blue area refers to the saline solution, and the orange area refers to the mixing area. The left image shows a saline solution droplet colliding a lipid ethanol solution droplet at negative $B$. The right one shows collision at not positive $B$. 
a)

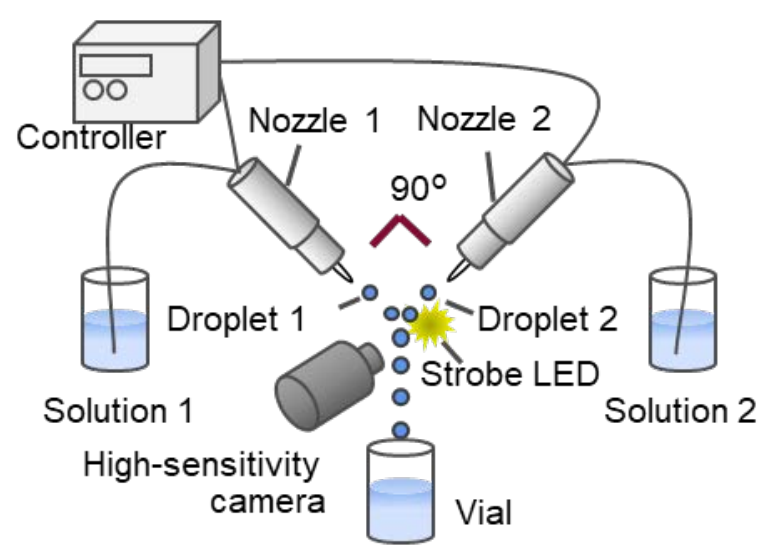

b)

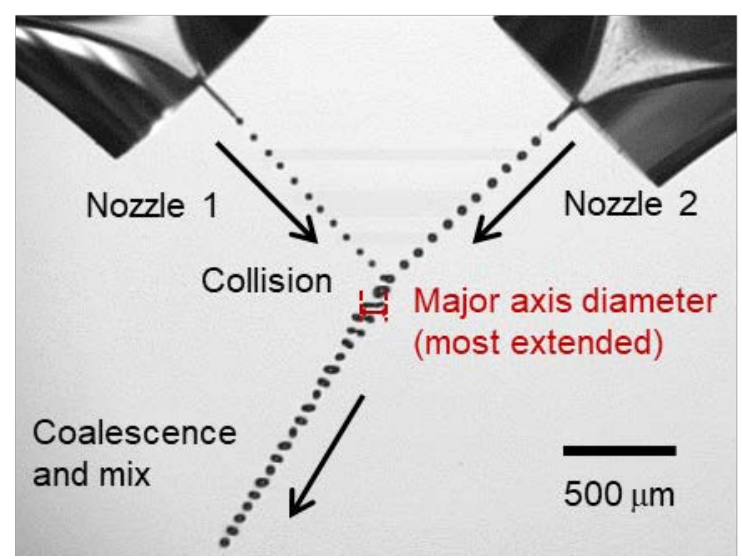


a) Relationship LNP size vs. We relative velocity of two droplets $u\left[\mathrm{~m} \mathrm{~s}^{-1}\right]$

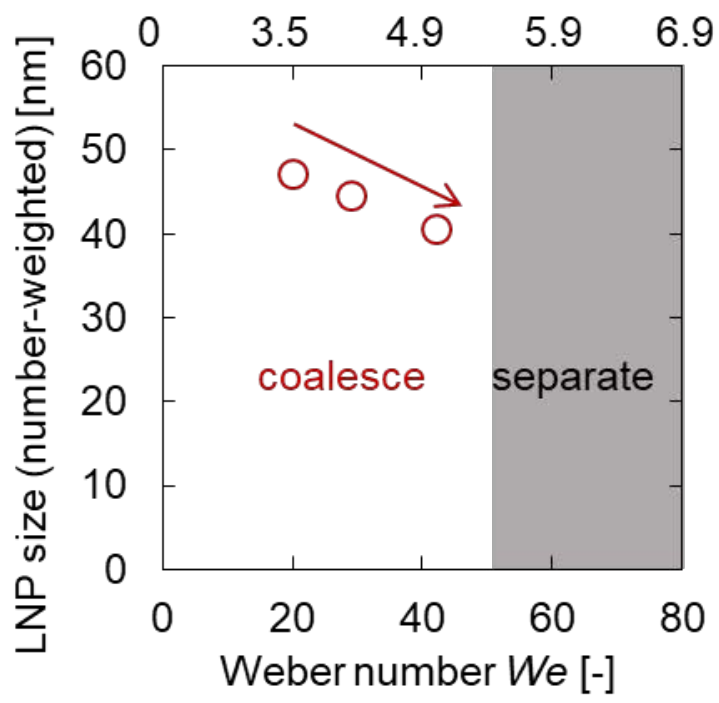

b) Particle size distribution (number-weighted)

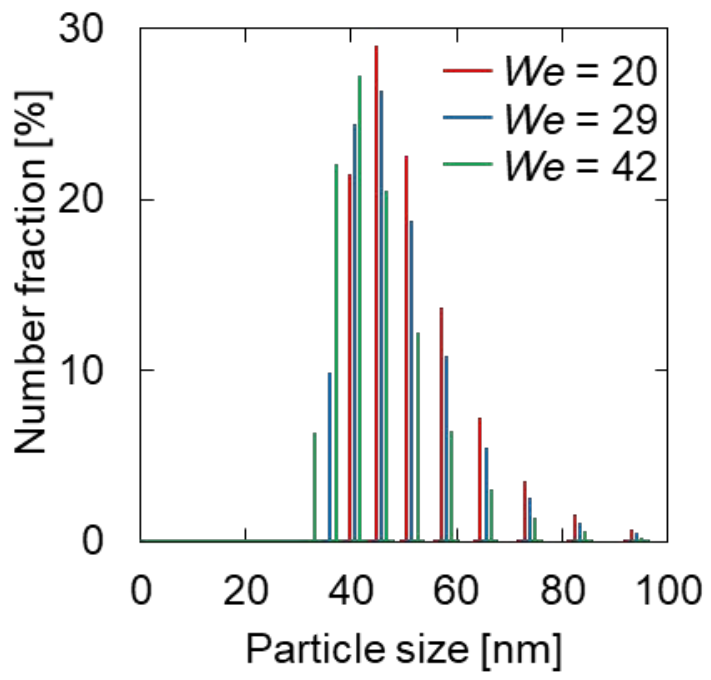

c) Deformation behavior of coalesced droplet

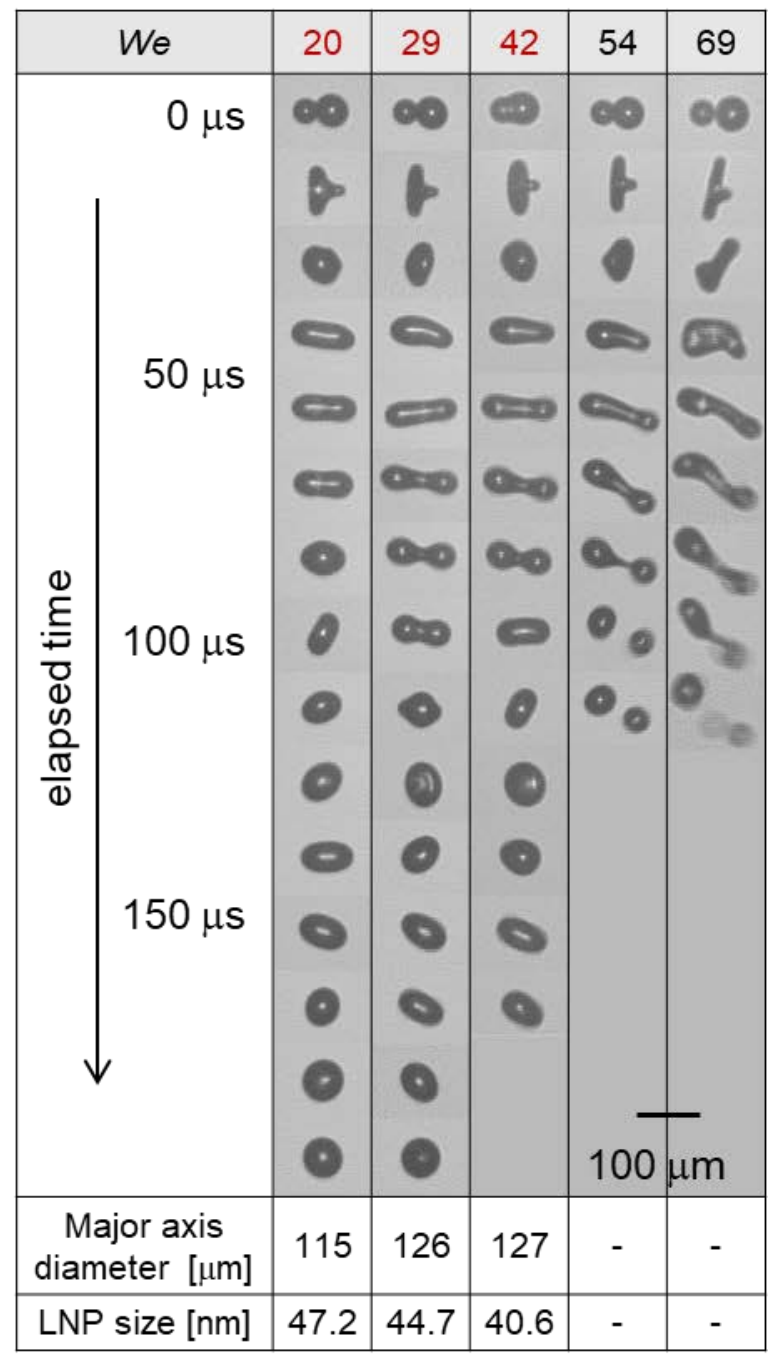


a) The impact parameters of condition i-vi
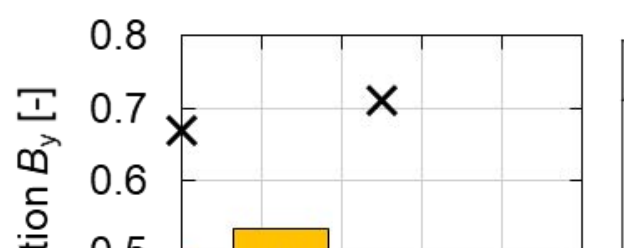

亭 0.5 d $\mathrm{i}$

. 0.4

产

음

윽

员

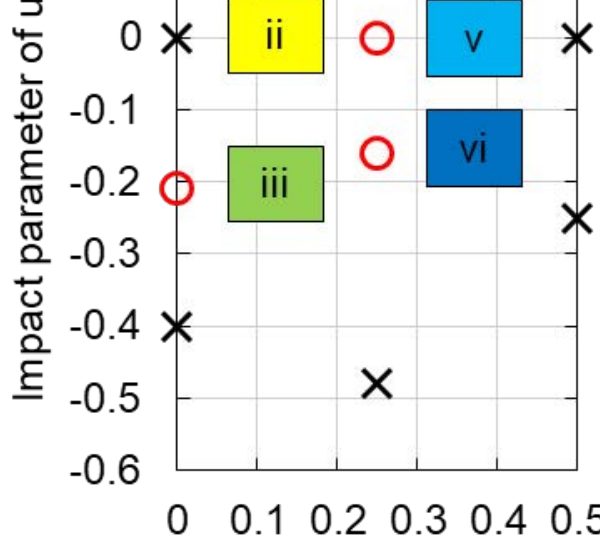

Impact parameter of depth direction $B_{\mathrm{z}}[-]$ b) Deformation behaviors of condition i-vi, most extended distance and LNP diameter each of them

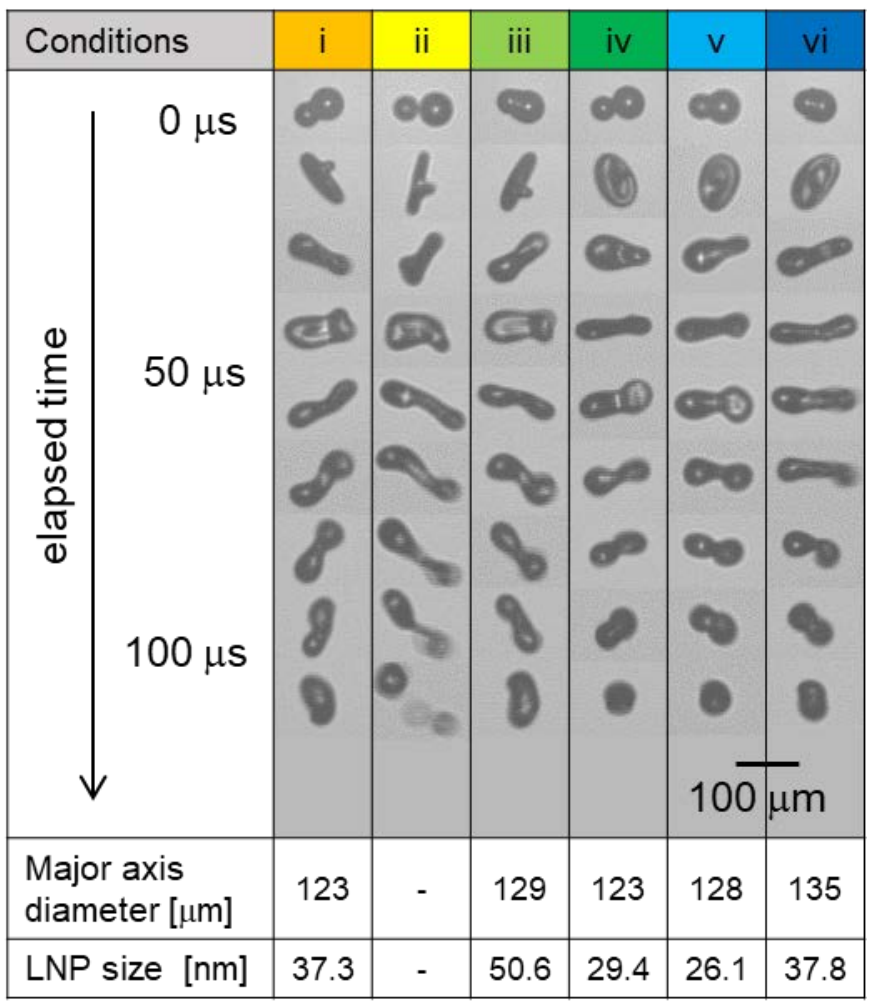




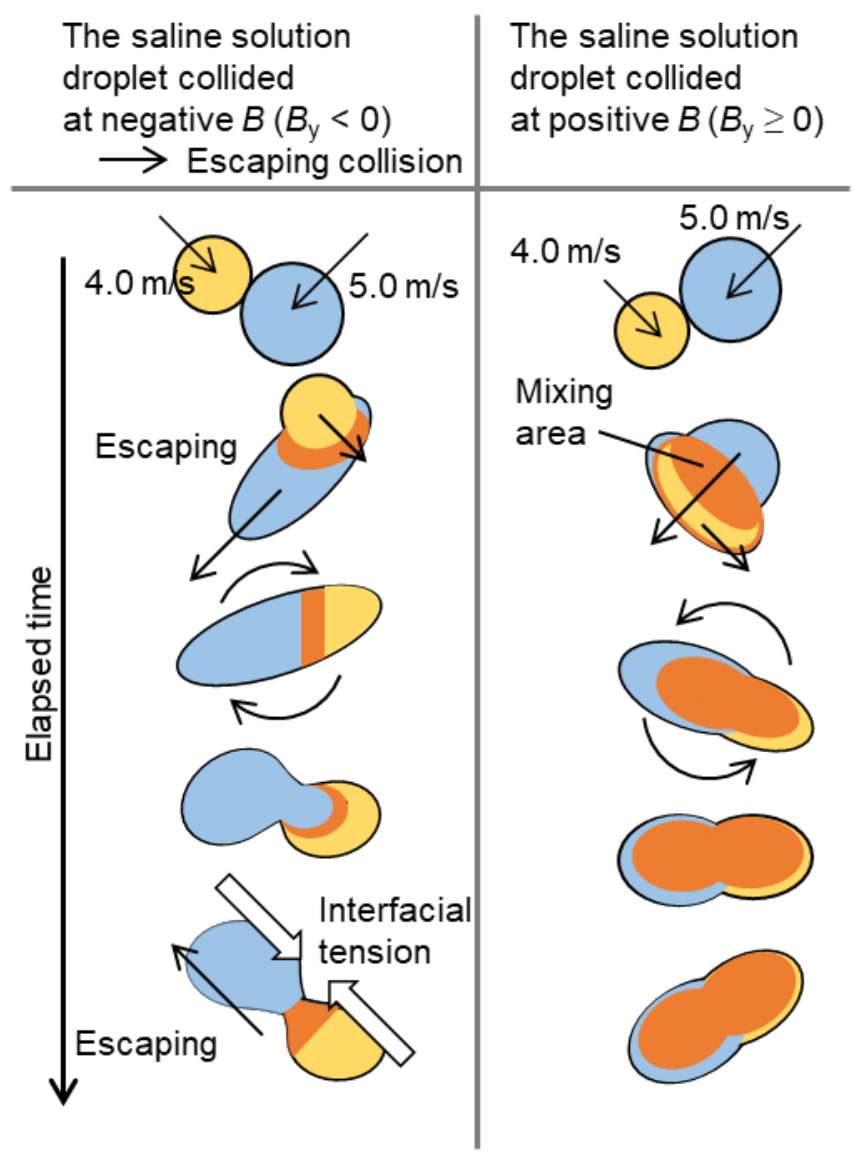




\section{Short text for the table of contents section}

An inkjet mixing system was investigated to synthesize lipid nanoparticles (LNPs). The system was employed to help minimize drug loss because the quick mixing of reagents enabled the synthesis of small LNPs with reducing excess amount of saline solutions which would contain drugs. And the system used minimal amount of reagent. Small LNPs were successfully synthesized. with less saline solutions.

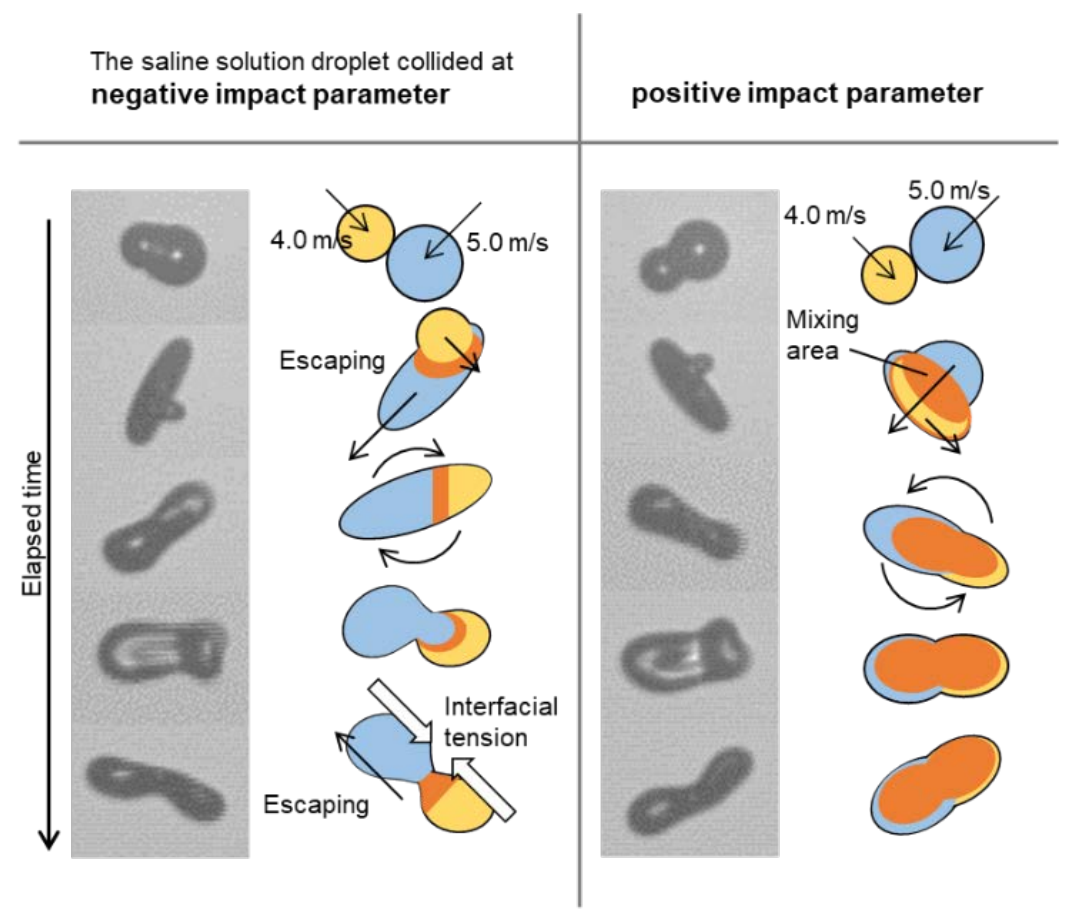

